\title{
Lineer Olmayan Dalga Denkleminin Lie Grupları Analizi
}

\author{
Lie Group Analysis of Nonlinear Wave Equation
}

\author{
Saadet ÖZER \\ İstanbul Teknik Üniversitesi, Fen Edebiyat Fakültesi, Matematik Mühendisliği Bölümü, Maslak 34469 İstanbul
}

Öz

Keyfi fonksiyonlar ya da parametreler içeren denklem kümesini, denklem ailesi olarak adlandırsak, ailenin üyeleri arasında geçişi mümkün kılan dönüşümler eşdeğerlik dönüşümleri olarak adlandırılır. Eşdeğerlik grupları, verilen bir diferansiyel denklem ailesini değişmez bırakan dönüşüm grupları olarak tanımlanır. Bu nedenle diferansiyel denklem ailelerinin eşdeğerlik grupları, aynı aileye ait, farklı denklemler arası mümkün ilişkilerin varlığını inceler ve bu ilişkileri ortaya çıkarır. Böylelikle, karmaşık bir denklemin tam çözümü ya da başka deyişle davranışı, aynı aileden daha basit bir denklem aracılığıyla belirlenebilir. Bu çalışmada, lineer olmayan tek boyutlu dalga denkleminin eşdeğerlik grupları, Lie gruplarının bir uygulaması çerçevesinde incelenmiş ve bazı örnekler ile lineer ve lineer olmayan denklemler arası geçişler sağlanmış, bazı karmaş1k lineer olmayan denklemlerin çözümü belirlenmiştir. Bu tipte dönüşümlerin varlığ için, sonsuz küçük üreteçler üzerine gelen şartlar elde edilmiştir. Ayrıca, bu şekilde nokta dönüşümleri aracılığı ile, lineer dalga denklemine dönüştürülebilen, lineer olmayan denklemlerin asgari fonksiyonel bağlılıkları da belirlenmiştir.

Anahtar Kelimeler: Eşdeğerlik Grupları, Lie Grupları, Dalga denklemi, Lineer olmayan denklemlerin tam çözümleri

\section{Abstract}

Differential equations involving some free functions or parameters of independent, dependent variables and their derivatives are called "family of differential equations". Each member of the family can represent the behavior of different materials for physical problems. Transformations which allow generating maps between members of the family, by keeping the family invariant are called equivalence transformations. Equivalence groups are defined as the group of transformations which leave a given family of differential equations invariant. Therefore, equivalence groups of family of differential equations may investigate the existence of possible relations between different equations belong to the same family and discover such relations. Equivalence groups of differential equations have been a great interest by the researchers not only to generate the invariant solutions or group classification of differential equations, but only to determine their exact solutions. If an admissible transformation exists, exact solution or in another word, behavior of a complicated equation can be determined via a simple equation from the same family by equivalence transformations.

In this work, the equivalence groups of nonlinear one dimensional wave equation are investigated within the framework of an application to Lie groups and especially, the existence of appropriate transformations between linear and proper nonlinear (quasilinear) equations are discussed in details. Exact solutions of some nonlinear equations are also obtained.

In the present paper, to investigate equivalence transformations, a general nonlinear one dimensional wave equation is taken in the form

$u_{t t}-f\left(x, t, u, u_{x}, u_{t}\right)_{x}=0$

as a family of equation, where $\mathrm{x}$ and $\mathrm{t}$ are the independent variables representing local coordinate and time, respectively, $\mathrm{f}$ is a continuously differentiable free function of its variables and subscripts denote the partial derivative with respect to related coordinates. A general vector field on the tangent space of the extended manifold can be given by

$V=X \frac{\partial}{\partial x}+T \frac{\partial}{\partial t}+U \frac{\partial}{\partial u}+V_{1} \frac{\partial}{\partial u_{x}}+V_{2} \frac{\partial}{\partial u_{t}}+S^{1} \frac{\partial}{\partial f}+S_{1}^{1} \frac{\partial}{\partial f_{x}}+S_{2}^{1} \frac{\partial}{\partial f_{t}}+S \frac{\partial}{\partial f_{u}}+S^{11} \frac{\partial}{\partial f_{u_{x}}}+S^{12} \frac{\partial}{\partial f_{u_{\mathrm{r}}}}$. 
To determine the equivalence groups, here we have used the method which is called Isovector method. It depends on the geometric approach to Lie groups generated by Harrison and Estabrook [27], developed then after by Edelen [29] and Şuhubi [29] for symmetry groups. Şuhubi [21] studied the method for equivalence groups and obtained the explicit solutions of the determining equations namely, components of isovector field for general second order Balance equations. Here we have used these results directly by expressing the wave equation as a balance equation.

The most general infinitesimal generators associated to the equivalence groups for the family of one dimensional wave equation are explicitly determined. We showed that if the infinitesimal generator $U$ which generates the transformation of the dependent variable, depends on the dependent variable $u$ nonlinearly, if the infinitesimal generator related to local coordinate $X$, depends on the dependent variable. Basically, $\boldsymbol{U}_{u u} \neq \mathbf{0}$ is possible if and only if $X_{u} \neq \mathbf{0}$. In addition to this result, the minimal functional dependence of the free function $f$ has been also investigated under some restrictions on it, so that transformations between linear and nonlinear members of the family of wave equation are possible. For that purpose, a particular restriction to the family has been taken by considering $\frac{\partial f}{\partial u_{t}}=\mathbf{0}$, namely, another family of wave equation is examined in the form of

$u_{t t}-f\left(x, t, u, u_{x}\right)_{x}=0$.

Due to the special functional form of $f$, as the variables $\boldsymbol{f}_{u_{t}}$ is missing from the extended manifold, the corresponding component of isovector field should vanish; $S^{12}=0$. We have showed that in this case, the theorem developed in the previous case is no longer valid. We also discovered that appropriate equivalence transformations between linear and nonlinear members of the wave equation taken in the form in this text, are only possible when the free function $f$ depends on $u_{t}$, in other words, a nonlinear one dimensional wave equation can be reduced into a linear one, if it involves $\boldsymbol{u}_{t}$.

In the last section, some examples of equivalence transformations between linear and nonlinear one dimensional wave equations are given. To show the reader how the procedure runs, for simplicity the constant coefficient wave equation $u_{x x}-u_{t t}=0$ $u_{x x}-u_{t t}=\mathbf{0}$ is taken into consideration by choosing $f=u_{x}$ . A special simple equivalence transformation: $\bar{x}=\boldsymbol{x}-\boldsymbol{\epsilon} \boldsymbol{u}$ is choosen as an example which maps the nonlinear equation

$2 \epsilon \bar{u}_{\bar{t}}\left(1+\epsilon \bar{u}_{\bar{x}}\right) \bar{u}_{\bar{x} \bar{t}}+\left(1-\epsilon^{2} \bar{u}_{\bar{t}}^{2}\right) \bar{u}_{\overline{x x}}-\left(1+\epsilon \bar{u}_{\bar{x}}\right)^{3} \bar{u}_{\bar{t} \bar{t}}=0$

onto the constant coefficient wave equation, so that the general solution of the wave equation $u=\Psi(t-x)+\Phi(t+x)$ generates $\bar{u}-\Psi(\bar{t}-\bar{x}-\epsilon \bar{u})-\boldsymbol{\Phi}(\bar{t}+\bar{x}+\epsilon \bar{u})=0$ as an implicit solution to the nonlinear equation. The infinitesimal generators obtained in this study can be used to map any member of the family, even the ones is not a wave equation. To show that, by taking $f=u$, the constant coefficient equation $u_{x}-u_{t t}=0$ is also studied as another example and mapped onto a nonlinear differential equation.
It is obviously clear, other types of transformations such as between constant coefficient and variable coefficient or homogenous and nonhomogeneous wave type equations, which can be expressed as members of family we have considered here can be investigated by choosing various different infinitesimal generators. In this paper, we have only concentrated on the transformations between linear and nonlinear wave equations as members of the particular family examined here.

Keywords: Equivalence Groups, Lie Groups, Wave equation, Exact solutions of nonlinear equations

\section{I.GiRiş}

Lineer olmayan diferansiyel denklemlerin çözümlerinin tam, yaklaşık ya da sayısal olarak belirlenmesi, çözümlerin belirlenmesinin mümkün olmadığı hallerde ise, denklemlerin davranışının incelenmesi diferansiyel denklemlerin en ilgi çeken, temel konularındandır. Bu amaçlarla, birçok araştırmacı tarafından çok değişik yöntemler, yaklaşımlar geliştirilmiş ve halen geliştirilmektedir. Lie gruplarının diferansiyel denklemlere uygulanması, tam çözümlerin belirlenmesi açısından uygulanan yöntemlerden biridir. Lie grupları, diferansiyel denklemlerin değişmez çözümlerinin elde edilmesi, denklemlerin sinıflandırılması, korunum yasalarının bulunması ve birbirine denk denklemlerin belirlenmesi açısından güçlü bir yaklaşımdır.

Yapısında keyfi parametre ya da fonksiyonlar barındıran denklemler, denklem ailesi olarak adlandırılabilir. Lie gruplarının diferansiyel denklemlere uygulanması çerçevesinde, simetri grupları bir denklemin çözüm ailelerini sınıflandırmak için kullanılır iken, eşdeğerlik grupları, denklem ailesinin yapısını korumakla birlikte, içerisindeki keyfi fonksiyonların değişimine izin verdiği için, uygun dönüşümler bulunabildiği takdirde davranışı bilinen bir denklem ile daha karmaşık olan bir diğeri arasında dönüşümlerin mümkün sınıflarını üretir. Bu anlamda, diferansiyel denklemlerin eşdeğerlik dönüşümleri, esasen, aynı aileye ait farklı denklemler arasındaki ilişkileri yapılandırır. Temel fizik ve mühendislik problemlerini temsil eden diferansiyel denklemler, problemin içerisinde bulunduğu ortamın temel özelliklerini ifade eden bazı parametrelerce birbirlerinden farklılaşır. Bu anlamda, özünde yapı korunur iken, yukarıda da ifade edildiği üzere, aynı denklem ailesinin farklı üyeleri, farklı malzemeler için farklı denklemlerce temsil edilir. Eşdeğerlik dönüşümlerinin gruplarının bu tip denklemler arası ilişkilerin yapılandırılmasında da kullanılabileceği öngörülebilir. Örnek verecek olursak, eşdeğerlik grupları, homojen ile homojen olmayan, sabit katsayılı ile değişken katsayılı denklemler arasında geçişi mümkün kılmasının yanı sıra, lineer ve lineer olmayan denklemler arasında dönüşümleri de 
sağlaması anlamında elverişlidir. Bu mümkün dönüşümler, denklemlerin çözümlerinin, davranışlarının belirlenmesinde de kullanılabilir. Özellikle lineer olmayan denklemlerin tam çözümlerinin belirlenmesinde önemli bir yaklaşım olarak ele alınabilir. Son yıllarda birçok araştırmacı tarafından lineer olmayan diferansiyel denklemlerin incelenmesinde, gerek değişmez çözümlerin bulunması [1-7], gerek denklemlerin sinıflandırılmasında [7-9], gerek se tam çözümlerin belirlenmesinde [10-14], eşdeğerlik grupları kullanılmıştır.

Eşdeğerlik Grupları hakkında genel bilgi [15-19] kaynaklarında bulunabilir. Lie 'nin klasik değişmezlik yaklaşımının eşdeğerlik dönüşümlerinin gruplarını üretmek için kullanılabileceği fikri ilk olarak Ovsiannikov [16]'a dayanır, sonrasında bu fikrin uygulanması anlamında değişik yöntemler geliştirilmiştir. Eşdeğerlik dönüşümlerinin belirlenmesi için kullanılabilecek yöntemler [17-19]'da ayrıntıları ile verilmiştir. Bu yöntemlerin dışında, bizim bu çalışmada da kullanacağımız, Şuhubi [20-24] tarafından geliştirilen, genel denklik denklemlerinin eşdeğerlik gruplarının izovektör alanı, bir başka deyişle, grubun sonsuz küçük üreteçlerinin çözümleri, diğer yöntemlere göre daha kısa ve kolay uygulanabilir bir yöntemdir.

Bu çalışmada tek boyutlu lineer olmayan dalga denklemleri ailesi ele alınmıştır. Lineer olmayan dalga denklemleri matematiksel fiziğin birçok alanında geniş uygulamaya sahip olmaları nedeni ile birçok alandan araştırmacı tarafından ele alınmış ve yaklaşık çözümler ya da sayısal çözümler dışında Lie grupları uygulamaları alanında da ilgi görmüştür. Lie grupları'nın dalga denklemlerine uygulamalarının oldukça geniş özeti Huang ve arkadaşlarının çalışmasından [25] incelenebilir.

En genel anlamda tek boyutlu dalga denklemi, Şuhubi [26] tarafından klasik izovektör yöntemi ile, denklemi dış formlar aracılığı ile yazarak ele alınmış, izovektör alanının üreteçleri belirlenmiş, bazı özel durumlar için üreteçlerin yapısı elde edilmiştir. Şuhubi, daha sonra bir başka çalışmasında [21], izovektör alanının katsayılarını, ikinci mertebeden genel denklik denklemleri için elde etmiş ve en genel dalga denklemini bir örnek olarak tekrar ele almıştır. Biz bu çalışma çerçevesinde, Şuhubi 'nin [26]'de ele aldığı özel bir hal ve onun daha ayrıntılı özel halini, [21] ile elde ettiği yönteme uygulayarak elde ettik ve problemi daha da ötesine taşıarak, lineer ve lineer olmayan denklemler arasında geçişleri mümkün kılan dönüşümlerin koşullarını elde edip, çeşitli örnekler üzerinden lineer olmayan denklemlere geçişler sağlayıp, bu denklemlerin tam çözümlerini elde ettik.

Okuyucuya, makalenin takibini kolay sağlamak anlamında özetleyecek olur isek, bu çalışma kapsamında esas olarak $u_{\mathrm{tt}}-f\left(x, t, u, u_{x}, u_{\mathrm{t}}\right)_{x}=0$

dalga tipi denklemlerin eşdeğerlik dönüşümlerinin yapısı incelenmiştir. Burada $x$ ve $t$ bağımsız değişkenleri, $u$ bağımlı değişkeni, alt indis, gösterdiği değişkene göre kısmi türevi, $f$ ise değişkenlerinin sabit olmayan bir fonksiyonunu temsil etmektedir. Temel bilgiler olarak adlandırılan 2. bölümde, genel tanımlar verildikten sonra, Şuhubi nin [21] çalışmasında elde ettiği, ikinci mertebeden denklik denklemlerinin izovektör alanınının katsayılarını açık olarak belirlediği yöntemin özeti verilmiş, yöntem, 3. bölümde yukarıda verilen dalga denklemine uygulanmıştır. Burada temel olarak lineer ve lineer olmayan denklemler arası dönüşümlerin mümkün yapıları irdelenmiş, elde edilen sonuç, Teorem 2 ile yazılmıştır. Daha sonra, yapının daraltılması ile oluşan farklılıklar tartışılmış ve (1) tipi denklem ailesi için, eşdeğerlik dönüşümleri aracılığı ile, lineer denklemlere indirgenebilen, lineer olmayan denklem ailelerinin fonksiyonel bağl11ıkları üzerine gelen kısıt Teorem 3 ile verilmiştir. 4 . bölümde ise, bir önceki bölümde elde edilen dönüşüm gruplarının alt gruplarından bazı örnekler ile, denklem ailesinin bazı üyeleri arasında, lineer ve lineer olmayan denklemler arası dönüşümler çalışılmış, karmaşık denklemlerin tam çözümleri, çözümü bilinen basit denklemler aracılığg ile belirlenmiştir. 5. ve son bölümde ise elde edilen sonuçlar tartışılmış ve çalışmanın ilerisi için bazı önerilerde bulunulmuştur.

\section{TEMEL BILGILER}

\section{I Temel Kavramlar}

Eğer bir diferansiyel denklem, içerisinde bulunduğu ortamın fiziksel özelliklerini yansıtan bir takım keyfi fonksiyon ya da parametreler içeriyor ise "denklem ailesi" olarak adland1rılır. Ailenin her bir üyesi yapısal olarak ana denklemin özelliklerin taşımak ile birlikte, özünde farklı diferansiyel denklemlerdir.

\section{Tanım I:}

$\left\{x_{i}, u^{\alpha}\right\}, i=1,2, \ldots n, \alpha=1,2, \ldots N \quad$ siras1 ile bağımlı, bağımsız değişkenlerin uzayını temsil etsin. $\Sigma_{k}\left(x_{i}, u^{\alpha}, u_{x_{1} x_{2} \ldots x_{\eta}}^{\alpha}\right), k=1,2, \ldots m$ ler, $u_{x_{1} x_{2} \ldots x_{n}}^{\alpha}$, bağıml değişkenlerin bağımsız değişkenlere göre keyfi mertebe türevlerini temsil edecek şekilde, değişkenlerinin keyfi fonksiyonları olsun. Bu halde,

$\Delta\left(x_{i}, u_{\alpha}, u_{x_{1} x_{2} \ldots x_{p}}^{\alpha}, \Sigma_{k, q}\left(x_{i}, u^{\alpha}, u_{x_{1} x_{2} \ldots x_{p}}^{\alpha}\right)\right)=0$ 
denklemi bir diferansiyel denklem ailesi olarak adlandırılır. Burada $\Sigma_{k, q}$ ile, $\Sigma_{k}$ fonksiyonu ve onun bağımlı, bağımsız değişkenler ve onların türevlerine göre kısmi türevleri temsil edilmektedir.

\section{Tanım 2:}

$\Delta\left(x_{i}, u_{\alpha}, u_{x_{1} x_{2} \ldots x_{i}}^{\alpha}, \Sigma_{k, q}\left(x_{i}, u^{\alpha},, u_{x_{1} x_{2} \ldots x_{p}}^{\alpha}\right)\right)=0$ ile verilen bir diferansiyel denklem ailesi için, $\varepsilon$ ile temsil edilen eşdeğerlik grubu, bağımlı, bağımsız değişkenler, onların türevlerinin, diferansiyel denklemin yapısını koruyan, fakat, ailenin herhangi

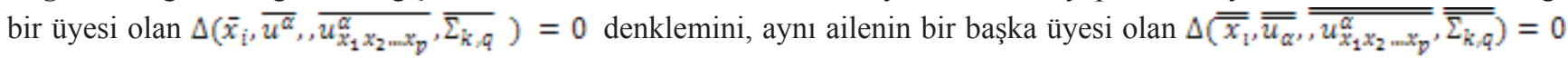
denklemine dönüştüren sürekli dönüşümlerin grubudur.

Daha anlaşılır olması için, bu çalışmada ele alınacak olan (1) ile verilen dalga denklemi üzerinde ifade edecek olursak, denklemin $\varepsilon$ eşdeğerlik grubu, açık hali ile aşağıda ifade edilen geçişi sağlayan dönüşümlerin grubudur:

$u_{\mathrm{tt}}-f\left(x, t, u, u_{x}, u_{\mathrm{t}}\right)_{x}=0 \Rightarrow \bar{u}_{\overline{t t}}-\bar{f}\left(\bar{x}, \bar{t}, \bar{u}, \bar{u}_{g}, \bar{u}_{\bar{t}}\right)_{x}=0$.

\section{Tanım 3:}

Genel bir ikinci mertebe denklik denklemi aşağıdaki gibi tanımlanır:

$\frac{\partial \Sigma^{i}\left(x^{j}, u, u_{i j}\right)}{\partial x^{i}}+\Sigma\left(x^{j}, u, u_{i j}\right)=0, \quad i=1,2, \ldots, n$

burada $x^{j} x^{j}$ ler bağımsız değişkenleri, $u$ bağımlı değişkeni ve $\Sigma^{i}, \Sigma$ lar ise bağımlı ve bağımsız değişkenler ile türevlerine bağl1, düzgün keyfi fonksiyonları temsil etmektedir. Açıktır ki tekrarlayan indisler üzerinde toplama uylaşımı mevcuttur, metin içerisinde aksi belirtilmedikçe, tekrarlayan indisler üzerinde toplama uylaşımı olduğu anlaşılmalıdır.

Açıkça görülmektedir ki, (3) ile verilen ikinci mertebe genel denklik denklemi, (2) ile tanımlanan bir denklem ailesidir. Denklik denklemleri, klasik fiziğin hemen hemen tüm denklemlerini, uygun eşlemeler altında temsil edebilmesi açısından önemli uygulama alanlarına sahiptir.

\subsection{Yöntem}

Bu çalışmada kullanılan yöntem, Harrsion ve Estabrook'un [26] geliştirdiği, esasen Cartan`ın [28] diferansiyel denklemlerin dış formlar aracılığı ile yazılması fikrine dayanan geometrik bir yaklaşımdır. Yöntem, daha sonra Edelen [29] tarafından denklik denklemlerinin simetri dönüşümleri için genişletilmiş ve Özer [22] tarafindan birinci mertebe denklik denklemlerine, Şuhubi [23] tarafından, ikinci mertebe denklik denklemlerine ve son olarak yine Şuhubi [24] tarafindan keyfi mertebe denklik denklemlerinin eşdeğerlik dönüşümlerinin üretilmesine genişletilmiş, üreteçler belirleyici denklemlerinin çözülmesi ile açik olarak elde edilmiştir.

Şimdi, (3) ile verilen ikinci mertebeden genel bir denklik denkleminin eşdeğerlik dönüşümlerinin grubunun belirlenebilmesi için Şuhubi [24] tarafından geliştirilen yöntemin özetini verelim.

$M=R^{n}$; örtüsü $\left(x^{i}\right)$ olan $n$ boyutlu katman, $G=R^{n} \times R ;\left(x^{i}, u\right)$ koordinat örtülü graf uzayı olsun. İkinci mertebeden denklik denkleminin eşdeğerlik gruplarını yapılandırabilmek için, katmanımıza,

$v_{i}=u_{, i}$

ile tanımlanan yeni değişken ile, $\Sigma^{i}$ ve $\Sigma$ ’nın kendilerinin ve onların fonksiyonel bağlılıklarını göz önüne alabilmek için, aşağıda açık olarak verilen, bağlı oldukları değişkenlere göre türevlerinin:

$$
s_{j}^{i}=\frac{\partial \Sigma^{1}}{\partial x^{j}}, \quad \sigma^{i}=\frac{\partial \Sigma^{1}}{\partial u}, \quad s^{i j}=\frac{\partial \Sigma^{1}}{\partial v^{j}}, \quad t_{i}=\frac{\partial \Sigma}{\partial x^{i}}, \quad \tau=\frac{\partial \Sigma}{\partial u}, \quad t^{i}=\frac{\partial \Sigma}{\partial v^{i}}
$$

bağımsız değişkenler gibi eklenmesi gerekir. Böylelikle oluşturulan genişletilmiş katmanın koordinat örtüsü 
$K=\left\{x^{i}, u, v_{i}, \Sigma^{i}, \Sigma, s_{j}^{i}, \sigma^{i}, s^{i j}, t_{i}, \tau, t^{i}\right\}$

olacaktır. $K$ katmanının teğet uzayında bir vektör alanı $V \in T(K) V \in T(K)$,

$V=X^{i} \frac{\partial}{\partial x^{i}}+U \frac{\partial}{\partial u}+V_{i} \frac{\partial}{\partial v_{i}}+S^{i} \frac{\partial}{\partial \Sigma^{i}}+\delta \frac{\partial}{\partial \Sigma}+S_{j}^{i} \frac{\partial}{\partial s_{j}^{i}}+\delta^{i} \frac{\partial}{\partial \sigma^{i}}+S^{i j} \frac{\partial}{\partial s^{i j}}+T_{i} \frac{\partial}{\partial t_{i}}+\mathcal{T} \frac{\partial}{\partial \tau}+T^{i} \frac{\partial}{\partial t^{i}}$

şeklinde yazılır ve (3) ile verilen ikinci mertebe denklik denkleminin izovektör alanı olarak adlandırılır. Denklik denkleminin eşdeğerlik dönüşümlerinin grupları ise, aşağıda verilen teorem aracılığı ile tanımlanır.

\section{Teorem I: [I]}

Bir $m$ boyutlu $M$ türetilebilir katmanı üzerinde bir $V$ vektör alanı

$V=v^{i}(\boldsymbol{x}) \frac{\partial}{\partial x^{i}}, p=\varphi^{-1}(\boldsymbol{x})$

ile verilmektedir. Burada $(\Omega, \varphi), p \in M$ olacak şekilde bir tasvirdır. Bir ${ }^{\gamma: I \rightarrow M}$ eğrisi ancak ve ancak ${ }^{i}(t)$ koordinat fonksiyonları $\mathrm{R}^{\mathrm{m}} \mathrm{de}$

$\frac{d x^{i}}{d t}=v^{i}(\boldsymbol{x}(t)), \quad i=1,2, \ldots, m$

adi türevli denklem takımının bir çözümü ise $V$ vektör alanının bir integral eğrisidir.

Bu halde (3) ile verilen 2. mertebe denklik denklemlerinin eşdeğerlik dönüşümleri, (5) izovektör alanı bileşenleri ile, yukarıda verilen teorem uyarınca,

$\frac{d \bar{x}^{1}}{d \epsilon}=X^{i}, \frac{d \bar{u}}{d \epsilon}=U, \frac{d \bar{\Sigma}^{1}}{d \epsilon}=S^{i}, \quad \frac{d \bar{\Sigma}}{d \epsilon}=\delta$

adi türevli denklem takımının,

$\bar{x}^{i}(0)=x^{i}, \quad \bar{u}(0)=u, \quad \bar{\Sigma}^{i}(0)=\Sigma^{i}, \quad \bar{\Sigma}(0)=\Sigma$

başlangıç koşulları altında çözülmesi ile belirlenir.

(5) ile verilen izovektör alanının katsayıları, bir başka deyişle eşdeğerlik dönüşümlerinin grubunun sonsuz küçük üreteçleri, belirleyici denklemlerinin tam çözümleri elde edilerek Şuhubi [24] tarafından aşağıdaki şekilde elde edilmiştir:

$X^{i}=-\phi^{i}\left(x^{j}, u\right), \quad U=U\left(x^{j}, u\right)$,

$V_{i}=D_{i} U+\left(D_{i} \phi^{j}\right) v_{j}, \quad S^{i}=\left(w+\frac{\partial \phi^{j}}{\partial u} v_{j}\right) \Sigma^{i}-\left(D_{j} \phi^{i}\right) \Sigma^{j}+\alpha^{i j} v_{j}+\beta^{i}$,

$$
\delta=\left(w+\frac{\partial \phi^{1}}{\partial u} v_{i}\right) \Sigma+\mathrm{D}_{\mathrm{i}} S^{\mathrm{i}}
$$

Burada $D_{i}=\frac{\partial}{\partial x^{i}}+v_{i} \frac{\partial}{\partial u}, \alpha^{i j}=-\alpha^{j i}$ dir ve $w, \alpha_{i j}$ ile $\beta^{i},\left(x^{i}, u\right)$ 'nun fonksiyonlarıdır.

Ayrıca, (5) izovektör alanında, eşdeğerlik grubu için gerekli olan, keyfi fonksiyonların fonksiyonel bağlılıklarını göz önüne almak için eklenen ek bileşenlere ait katsayılar ise,

$S_{j}^{i}=\frac{\partial F^{1}}{\partial x^{j}}+\frac{\partial F^{1}}{\partial \Sigma^{k}} s_{j}^{k}+\frac{\partial F^{1}}{\partial \Sigma} t_{j}, \quad \delta^{i}=\frac{\partial F^{1}}{\partial u}+\frac{\partial F^{1}}{\partial \Sigma^{k}} \sigma^{k}+\frac{\partial F^{1}}{\partial \Sigma} \tau, \quad S^{i j}=\frac{\partial F^{1}}{\partial v^{j}}+\frac{\partial F^{1}}{\partial \Sigma^{k}} s^{k j}+\frac{\partial F^{1}}{\partial \Sigma} t^{j}$, 


$$
\begin{aligned}
& T_{i}=\frac{\partial G}{\partial x^{i}}+\frac{\partial G}{\partial \Sigma^{j}} s_{i}^{j}+\frac{\partial G}{\partial \Sigma} t_{i}, \quad \mathcal{T}=\frac{\partial G}{\partial u}+\frac{\partial G}{\partial \Sigma^{j}} \sigma^{j}+\frac{\partial G}{\partial \Sigma} \tau, T^{i}=\frac{\partial G}{\partial v^{i}}+\frac{\partial G}{\partial \Sigma^{j}} s^{j i}+\frac{\partial G}{\partial \Sigma} t^{i} \\
& F^{i}=-s_{j}^{i} X^{j}-\sigma^{i} U-s^{i j} V_{j}+S^{i}, \quad G=-t_{i} X^{i}-\tau U-t^{i} V_{i}+\delta
\end{aligned}
$$

eşitlikleri ile belirlenir. Bu katsayılar genel ifadelerdir, ele alınan diferansiyel denklemin yapısı gereği $\Sigma^{i}$ ler ile $\Sigma$ 'nın bazı değişkenlere bağlılıkları olmaması halinde, onların katsayılarının izovektör alanında sıfıra özdeş olması gerekir. Öte yandan $\Sigma^{i}$ ler ile $\Sigma$ 'nın gerek birbirleri, gerek se genişletilmiş katmanın koordinat örtüsünün elemanları arasında bazı ilişkiler olması halinde, bu ilişkiler aracılığı ile vektör alanının katsayıları arasında da bir takım ilişkiler olacaktır. Bir sonraki kısımda burada özetlenen yöntem, (1) ile verilen tek boyutlu dalga tipi denklemine uygulanacak ve eşdeğerlik dönüşümlerini üretecek olan izovektör alanı katsayıları, başka deyişle, dönüşüm gruplarının sonsuz küçük üreteçleri açık olarak elde edilecektir.

\section{Tek Boyutlu Dalga Denkleminin Eşdeğerlik Gruplarının Sonsuz Küçük Üreteçleri}

(1) denklemi ile verilen dalga denklemini (3) ile yazılan denklik denklemine eşleyebilmek için aşağıdaki tanımlamaları yapalım:

$x^{1}=x, x^{2}=t, v_{1}=u_{x}, v_{2}=u_{\mathrm{t}}, \quad \Sigma^{1}=f, \Sigma^{2}=-v_{2}, \Sigma=0$.

Dolayısıyla ele alacağımız denklik denklemi, özel halde

$\frac{\partial f\left(x^{1}, x^{2}, u, v_{1}, v_{2}\right)}{\partial x^{1}}-\frac{\partial v_{2}}{\partial x^{2}}=0$

olacaktır. Bu halde (5) ile verilen izovektör alanına eş izovektör alanı, (1) denklemi için

$V=X \frac{\partial}{\partial x}+T \frac{\partial}{\partial t}+U \frac{\partial}{\partial u}+V_{1} \frac{\partial}{\partial v_{1}}+V_{2} \frac{\partial}{\partial v_{2}}+S^{1} \frac{\partial}{\partial f}+\cdots$

olarak yazılır. Öte yandan (4) ile tanımlanmış olan ek değişkenlerden sıfırdan farklı olanlar, (8) denklikleri göz önünde bulundurularak aşağıdaki gibi elde edilir:

$s_{1}^{1}=f_{x}, \quad s_{2}^{1}=f_{\mathrm{t}}, \quad \sigma^{1}=f_{u}, \quad s^{11}=f_{u_{x^{\prime}}}, \quad s^{12}=f_{u_{\mathrm{e}}}, \quad s^{22}=-1$.

Ayrıca, sıfira denk olan bileşenler ise,

$$
s_{1}^{2}=s_{2}^{2}=\sigma^{2}=s^{21}=t_{1}=t_{2}=t^{1}=t^{2}=\tau=0 s_{1}^{2}=s_{2}^{2}=\sigma^{2}=s^{21}=t_{1}=t_{2}=t^{1}=t^{2}=\tau=0
$$

olacaktır.

Uygulamada genellikle, lineer ve lineer olmayan diferansiyel denklemler arası geçişler, bağımlı değişkenin izovektör alanı katsayısının lineer olmayan bir yapıda alınması ile sağlanır. Fakat (1) denklem ailesinin üyeleri arasında bu durumda önemli bir kısıt ortaya çıkmaktadır. Aşağıdaki Teorem, (1) ile ifade edilen dalga denklemleri ailesinin, lineer ve lineer olmayan üyeleri arasında geçiş sağlayacak mümkün dönüşümlerin üretilmesinde kullanılacak olan sonsuz küçük üreteçlerin sağlaması gereken asgari koşulları belirler.

\section{Teorem 2:}

$u_{t t}-f\left(x, t, u, u_{x}, u_{t}\right)_{x}=0$ dalga denklemi ailesinin eşdeğerlik dönüşümlerinin yapısında, bağımlı değişkenin dönüşümünün sonsuz küçük üreteci olan $U$, ancak yerel koordinatın dönüşümünün sonsuz küçük üreteci $X$ in, bağımlı değişken olan $u$ ya bağlı olması halinde, bağımlı değişkene lineer olmayan bir bağlılığa sahip olabilir. 


\section{İspat:}

(1) ile verilen dalga denklemi ailesinin eşdeğerlik dönüşümlerinin grupları, (9) izovektör alanı katsayıları ya da diğer adı ile dönüşümlerin sonsuz küçük üreteçlerinin, yukarıda (8) ile ifade edilen denkliklerin ve (10) ve (11) ile verilen ek değişkenlerin üreteceği kısıtların, $(6) \mathrm{v}_{s}^{-2}$ ) denkle $\sum_{\Sigma}$ lerine yazılıp uygun düzenlemeler ile belirlenmesi ile elde edilir. Öncelikle, açıktır ki (11) ek değişkenlerinin, ’nin ve 'nın vektör alanındaki katsayıları özdeş olarak sıfır olmalıdır:

$$
\begin{aligned}
& S_{1}^{2}=S_{2}^{2}=\delta^{2}=S^{21}=T_{1}=T_{2}=T^{1}=T^{2}=\mathcal{T}=S^{22}=0 \\
& \delta=0
\end{aligned}
$$

Ayrıca, açıkça görülür ki (8) denklikleri nedeni ile (5) ve (9) izovektör alanı katsayıları arasında

$$
S^{2}+V_{2}=0
$$

iliş̧kisi vardır. (12) özdeşliklerini (7) denklemlerinden hesaplamak için, öncelikle ${ }^{F^{1}, F^{2}}$ ve $G$ belirlenmelidir. ${ }^{G=0}$ olduğu çok açıktır, $\mathrm{do}_{F^{2}}^{1}={ }_{0}^{-1} \mathrm{e}(12)$ eşitliklerinden, $G$ ye bağlı olan tüm ek izovektör katsayıları otomatik olarak sıfira denk olacaktır. Aynı şekilde olduğundan, ona bağlı ek bileşenlerin de özdeş olarak sıfira eşit olmasından herhangi bir ek kısıt üretilmeyecektir. Bu durumda, eşdeğerlik dönüşümlerinin gruplarının yapısı, ancak geriye kalan (13) ve $\left.{ }_{S}^{1} 14\right) \mathrm{d}_{\widehat{V}_{2}}$ klemleri ile elde edilecek olan belirleyici denklemlerin çözümü ile belirlenecektir. (14) denklemi, (6) eşitliklerinden ve ve 'nin düzenlenmesi ile açık olarak yazıldığı zaman elde edilen denklem, $K$ katmanının koordinat örtüsünün tüm bileşenlerinin bağımsız bileşenler gibi göz önüne alınması ile çözülür. Denklem bileşenlerine göre düzenlenirse

$-\left(\phi_{x}^{1}+\phi_{u}^{2} v_{1}\right) \Sigma^{1}+\left(\alpha^{21}+\phi_{t}^{1}\right) v_{1}+\left(U_{u}+2 \phi_{t}^{2}+\alpha^{22}-w\right) v_{2}+\phi_{u}^{2} v_{2}^{2}+\beta^{2}+U_{t}=0$

elde edilir. Burada tipik bir polinom özdeşliğinin çözümü ile

$\phi_{u}^{2}=\phi_{x}^{2}=0, \quad \alpha^{21}=-\phi_{t}^{1}, \quad w=U_{u}+\phi_{t}^{2}+\alpha^{22}, \quad \beta^{2}=-U_{t}$

kısıtları üretilir. Bu kısıtların kullanılması ile, bağımlı ve bağımsız değişkenlerin izovektör alanı katsayıları, yani, dönüşümlerinin grubunun sonsuz küçük üreteçlerinin yapısı

$X=-\phi^{1}(x, t, u), T=-\phi^{2}(t), U=U(x, t, u)$

olur. İzovektör alanının diğer bileşenlerinin katsayıları ise;

$V_{1}=U_{x}+\left(U_{u}+\phi_{x}^{1}\right) v_{1}+\phi_{u}^{1} v_{1}^{2}, \quad V_{2}=U_{t}+\phi_{t}^{1} v_{1}+\left(U_{u}+\dot{\phi}^{2}\right) v_{2}+\phi_{u}^{1} v_{1} v_{2}$

$S^{1}=\left(U_{u}+2 \phi^{2}+\alpha^{22}(x, t, u)-\phi_{x}^{1}\right) \Sigma^{1}+\alpha^{11}(x, t, u) v_{1}+2 \phi_{t}^{1} v_{2}+\phi_{u}^{1} v_{2}^{2}+\beta^{1}(x, t, u)$

formlarına indirgenir. Dikkat edilir ise burada, bağımlı ve ba sında herhangi bir kısıt bulunmamaktadır. Fakat (13) ile verilen denklemi sisteme yeni kısıtlar getirecektir. (6) eşitliklerinin sonuncusundan, denklem

$\frac{\partial S^{1}}{\partial x}+\frac{\partial S^{1}}{\partial u} v_{1}-\frac{\partial V_{2}}{\partial t}-\frac{\partial V_{2}}{\partial u} v_{2}=0$

şeklinde yazılır. Denklemde, ${ }^{1} v^{V_{2}}$ 'nin açık ifadesi (17) ve (16) eşitliklerinden yerine yazılıp, düzenlendikten sonra, izovektör bileşenlerinin katsayıları arasında aşağıda verilen ilişkilerin olması gerektiği görülür:

$\left(U_{u}-\phi_{x}^{1}\right)_{u}=0$,

$\left(U_{u}-\phi_{x}^{1}+\alpha^{22}(x, t)\right)_{x}=0, \quad\left(U_{u}-\phi_{x}^{1}\right)_{t}=-\frac{1}{2} \phi^{2}$ 
(18) denkleminden $U_{u u} \neq 0$ olması için, yani $U$ nun $u^{\prime}$ ya lineer olmayan bağl1lı̆̆ için $\phi^{1}$, in de bağımlı değişkene bağlı olması gerektiği görülür ki Teoremin ispatı burada tamamlanır.

Teoremin ispatında ele alınan son denklem, (18) ve (19) kısitlarına ek olarak

$\beta_{x}^{1}=U_{\mathrm{tt}}, \quad \alpha^{11}=\alpha^{11}(x, t), \quad \alpha^{22}=\lambda(x)+\gamma(t)$

denkliklerini de üretir. Burada ara işlemler aşikar olduğu için atlanacak, sadece elde edilen sonuçlar verilip, yorumlanacaktır. Tüm bu elde edilen kısıtlar değerlendirilerek (15-17) sonuz küçük üreteçleri açık olarak aşağıdaki gibi belirlenir:

$X=-\phi^{1}(x, t, u), T=-\phi^{2}(t), \quad U=\int \phi_{x}^{1} d u+\left(\lambda(x)-\frac{1}{2} \phi^{2}\right) u+\eta(x, t)$,

$V_{1}=\int \phi_{x x}^{1} d u+\hat{\lambda}(x) u+\frac{\partial \eta}{\partial x}+\left(2 \phi_{x}^{1}-\frac{1}{2} \dot{\phi}^{2}+\lambda(x)\right) v_{2}+\phi_{u}^{1} v_{1}^{2}$

$V_{2}=\int \phi_{x t}^{1} d u-\frac{1}{2} \ddot{\phi}^{2} u+\frac{\partial \eta}{\partial t}+\phi_{t}^{1} v_{1}+\left(\phi_{x}^{1}+\frac{1}{2} \dot{\phi}^{2}+\lambda(x)\right) v_{2}+\phi_{u}^{1} v_{1} v_{2}$

$S^{1}=\left(\frac{3}{2} \dot{\phi}^{2}+2 \lambda(x)+\gamma(t)\right) \Sigma^{1}+\alpha^{11}(x, t) v_{1}+2 \phi_{t}^{1} v_{2}+\phi_{u}^{1} v_{2}^{2}+\beta^{1}(x, t, u)$

burada $^{\beta_{x}^{1}=U_{t t}}$ eşitliğinin sağlanması gerektiği unutulmamalıdır. Eşdeğerlik dönüşümleri, yukarıda (20-23) ile belirlenmiş olan sonsuz küçük üreteçleri kullanılarak

$\frac{d \bar{x}}{d \epsilon}=X, \frac{d \bar{t}}{d \epsilon}=T, \frac{d \bar{u}}{d \epsilon}=U, \frac{d \bar{f}}{d \epsilon}=S^{1}$

denklem sisteminin

$\bar{x}(0)=x, \quad \bar{t}(0)=t, \quad \bar{u}(0)=u, \quad \bar{f}(0)=f$

başlangıç koşulları altında integre edilmesi ile belirlenir. (20-23) ifadelerinde geçen fonksiyonların integre edilebilir oldukları açıktır, fakat bu elbette ki keyfi fonksiyonlar ile bu integrallerin hesaplanabileceği anlamına gelmez. Bu nedenle, eşdeğerlik dönüşümlerini en genel yapıda elde etmek mümkün değildir. 4. bölümde fonksiyonların bir takım özel halleri ele al1narak, denklem sistemi integre edilecek ve bazı alt grupların eşdeğerlik dönüşümlerine örnekler verilecektir.

Tek boyutlu birinci mertebe dalga denklemi ailesi (1) 'in bazı alt aileleri için çalışma, burada elde edilen sonuçlar üzerine, denklemin yapısal özelliklerinin getireceği ek koşullar incelenerek kolaylıkla belirlenebilir. Aşağıda buna bir örnek olarak, bir özel hal ele alınacaktır.

\section{Teorem 3.}

$u_{\mathrm{tt}}-f\left(x, t, u, u_{x}, u_{\mathrm{t}}\right)_{x}=0 \quad$ şeklinde verilen dalga tipi denklem ailesinin,

$u_{\mathrm{tt}}+A(x, t) u_{x x}+B(x, t) u_{x t}+C(x, t) u_{x}+D(x, t) u_{t}+F(x, t)=0$

şeklinde bir lineer üyesi ile, ancak 
$\bar{u}_{\overline{t t}}-\bar{f}\left(\bar{x}, \bar{t}, \bar{u}, \bar{u}_{x}, \bar{u}_{\bar{t}}\right)_{x}=0, \quad \frac{\partial \bar{f}}{\partial \bar{u}_{\bar{f}}} \neq 0$

şeklinde bir lineer olmayan üyesi arasında, bir nokta tipi eşdeğerlik dönüşümü mümkündür.

\section{İspat:}

(1) ile ele alınan dalga tipi denklem ailesinin bir alt ailesi olarak, $f$ fonksiyonunu daha dar bir yapıda, ${ }^{u_{t}}$ ye bağlı olmayan halde ele alacak olursak, $f$ fonksiyonunun bu şekilde seçilmesi ile, denklem ailesi

$f\left(x, t, u, u_{x}\right)_{x}=u_{t t}$

olacaktır. (27) denklem ailesinin bir üyesi elbette (1) denkleminin de bir üyesidir, fakat (27) ailesinin eşdeğerlik gruplarının (1) ailesinin eşdeğerlik gruplarından daha dar bir yapıda olacağı kolaylıkla öngörülebilir. Bu noktada yapının daraltılmasının, mümkün eşdeğerlik dönüşümlerinde de bir daralmaya neden olacağı söylenebilir. O halde denklem ailesinin üyeleri arasında en geniş geçiş sağlayan dönüşümler nasıl belirlenebilir ve bu geçişler uygulama anlamına ne kadar elverişlidir sorusu akla gelir.

Şimdi (27) denklemin, (1) denkleminin eşdeğerlik grupları yapısı üzerine getirdiği kısıtları inceleyelim. (27) denkleminin yapısındaki fonksiyonel bağlılık nedeni ile ek değişkenlerden (11) de bulunan

$s^{12}=0$

olacağı, dolayısı ile ona bağlı izovektör alanı bileşeni

$S^{12}=0$

olması gerektiği açıktır. $^{S^{12}}$ 'nin (7) denkleminde verilen ifadesi yazılır ve fonksiyonel bağlılıklar dikkate alınır ise aşağıdaki iki denklem elde edilir:

$\frac{\partial V_{1}}{\partial v_{2}}=0, \quad \frac{\partial S^{1}}{\partial v_{2}}=0$.

İkinci denklemden, ${ }^{1}$ in (23) ile elde edilmiş olan ifadesi kullanılarak,

$\phi_{u}^{1}=0, \quad \phi_{t}^{1}=0$

sonuçları elde edilir ki Teorem 2`nin geçerliliğini kaybettiği görülür. Bu noktada aklan gelen soru, lineer ve lineer olmayan denklemler arası geçişin, böylesi bir yapıda hala geçerli olup olmadığıdır. Şimdi bu sorunun cevabını araştırmak için diğer üreteçleri belirleyelim. (18) denkleminden açıkça görülür ki, bu halde

$U_{\text {uu }}=0$

olacaktır. Dolayısı ile denklem ailesinin dönüşümünü kontrol eden üreteçler, yerel koordinatların, başka deyişle bağımsız değişkenlerin bağımlı değişkene bağlılığı yapısını ortadan kaldırmanın ötesinde, bağımlı değişkenin lineer olmayan dönüşümüne de imkan vermemektedir. Dolayısı ile buradan ancak lineer denklemlerin başka lineer de $u_{t}^{-1}$ lemlere dönüşebileceği sonucu çıkartılabilir, yapısal olarak lineer ve lineer olmayan denkle $\tilde{u}_{t}^{-}$ler arası geçiş, ancak $f$ nin ${ }^{u_{t}}$ 'ye bağlılığı ile mümkündür. Bu sonuç, lineer olmayan denklemde, lineer olmayan kısmın ${ }^{{ }^{t}}$ 'ye bağlı olacağı öngörüsünü ortaya çıkarır ki, teoremin ispatı burada tamamlanır.

Bir sonraki bölümde ele alınan örnekler ile okuyucu Teorem 3 ün sağlamasını görecektir. 


\section{TEK BOYUTLU DALGA DENKLEMININ EŞDEĞERLIK DÖNÜŞÜMLERININ LINEER OLMAYAN DENKLEMLERE UYGULAMALARI}

Bu bölümde, (1) ile verilen dalga denklemi ailesinin, eşdeğerlik dönüşümlerinin gruplarının, bazı alt grupları ele alınarak, denklem ailesinin iki üyesi arasında geçişler örneklenecektir. Çalışmanın amacı çerçevesinde, sadece lineer ve lineer olmayan denklemler arası dönüşümlere uygulamalar ele alınacaktır. Okuyucu ister ise benzer şekilde daha farklı tipte denklemler arası dönüşümleri, kolaylıkla üretebilir.

Örneklerde, (20-23) sonsuz küçük üreteçlerinin özel bazı halleri ele alınarak, (24) denklem sisteminin (25) başlangıç koşulları altında integre edilmesi ile dönüşümler belirlenip, geçişin ürettiği denklem ailesi üyeleri belirlenecek ve bu şekilde, lineer bir denklem için bilinen çözümden, eşdeğerlik dönüşümünün mümkün kıldığı karmaşık bir lineer olmayan denklemin tam çözümü bulunacaktır.

Örnek 1: Yukarıda (20-23) ile verilen sonsuz küçük üreteçlere bir örnek olarak

$\phi^{1}=u, \quad \phi^{2}=0$

alalım. Kolaylık olması açısından birbirleri ile ilişkileri de dikkate alacak şekilde, üreteçlerdeki serbest fonksiyonların tamamını özdeş olarak sıfır seçelim: $\alpha^{11}=\lambda=\gamma=\eta=0 \alpha^{11}=\lambda=\gamma=\eta=0$, bu halde

$U=0, \quad V_{1}=v_{1}^{2}, \quad V_{2}=v_{1} v_{2}, \quad S^{1}=-v_{1} v_{2}$

olacaktır. Bu ifadeleri (24) denklem sistemine yazıp, (25) başlangıç koşulları altında integre eder isek, alt grubun eşdeğerlik dönüşümleri aşağıdaki gibi belirlenir:

$\bar{x}=x-\varepsilon u, \bar{t}=t, \bar{u}=u$

$\bar{v}_{1}=\frac{v_{1}}{1-\epsilon v_{1}}, \quad \bar{v}_{2}=\frac{v_{2}}{1-\epsilon v_{1}}, \quad \bar{f}=f+\frac{\epsilon v_{2}^{2}}{1-\epsilon v_{1}}$.

Şimdi (1) denkleminde $f=u$ alalım. Bu durumda (1) ailesinin

$u_{x}-u_{t t}=0$

üyesi yazılır. Her ne kadar bu denklem, uzay zaman koordinatları yer değiştirmiş 1sı denklemi yapısında olup, dalga denk-

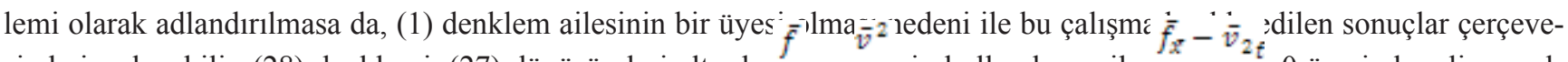
sinde incelenebilir. (28) denklemi, (27) dönüşümleri altında ve nin kullanılması ile ${ }^{2}=0$ üzerinden, lineer olmayan

$\left(1+\epsilon \bar{u}_{R}\right)\left(\left(\bar{u}_{R}-\bar{u}_{\mathrm{fz}}\right)\left(1+\epsilon \bar{u}_{R}\right)+2 \epsilon \bar{u}_{\tilde{t}} \bar{u}_{R t}\right)-\epsilon^{2} \bar{u}_{\tilde{t}}^{2} \bar{u}_{R X}=0$

denklemine dönüşür. Sabit katsayılı (28) denkleminin bir çözümü de (27), 'den elde edilen ters dönüşümler ile (29) denkleminin bir çözümünü üretir. Burada dikkat edilmesi gereken nokta, genel çözümlerin dönüşümler altında genel çözümler üretmediği, fakat lineer olmayan denklemi sağlayan fonksiyonlar ürettiğidir, bir başka deyişle, lineer olmayan denklemin bir tam çözümü yazılmış olur.

Şimdi okuyucunun hesapları sağlayabilmesi adına (28) denklemini sağlayan basit bir fonksiyon alalım:

$u(x, t)=\frac{1}{2} t^{2}+x$

(27) 'den elde edilen ters dönüşümler ile 
$\bar{u}=\frac{1}{(1-\epsilon)}\left(\frac{1}{2} \bar{t}^{2}+\bar{x}\right)$

fonksiyonunun, son derece karmaşık görünen (29) denkleminin bir açık çözümü olduğu kolaylıkla sağlanabilir.

Örnek 2: Bir önceki örnekte ele alınan dönüşümü bu kez $f=u_{x}$ seçerek klasik sabit katsayılı dalga denklemine uygulayalım:

$u_{x x}-u_{t t}=0$.

Bu durumda dönüşmüş haldeki denklem, gerekli hesaplar yapildiktan sonra

$2 \epsilon \bar{u}_{\bar{t}}\left(1+\epsilon \bar{u}_{\Omega}\right) \bar{u}_{X f}+\left(1-\epsilon^{2} \bar{u}_{f}^{2}\right) \bar{u}_{X X}-\left(1+\epsilon \bar{u}_{X}\right)^{3} \bar{u}_{f \epsilon}=0$

kuazilineer denklemine dönüşür. (30) dalga denkleminin genel çözümü olan $u=\Psi(t-x)+\Phi(t+x)$ $u=\Psi(t-x)+\Phi(t+x)$ fonksiyonu, yukarıdaki lineer olmayan denklem için

$\bar{u}-\Psi(\overline{\mathrm{t}}-\bar{x}-\epsilon \bar{u})-\Phi(\bar{t}+\bar{x}+\epsilon \bar{u})=0$

kapalı fonksiyonunu çözüm olarak üretir.

Sonsuz küçük üreteçlerin, her farklı seçiminde, farklı bir eşdeğerlik grubu altında yapının incelenebileceği açıktır. Lineer ve lineer olmayan denklemler dışında, sabit katsayılı ile değişken katsayılı ya da homojen ve homojen olmayan denklemler arasında da dönüşümler üretilebilir. Fakat bu çalışma kapsamında lineer olmayan denklemlerin, Lie grubunun bir uygulaması olan eşdeğerlik dönüşümleri grubu ile, çözümlerinin belirlenmesi konusu ele alınmıştır.

\section{SONUÇ VE YORUMLAR}

Çalışma kapsamında, (1) ile verilen tek boyutlu dalga tipi denklem ailesinin eşdeğerlik dönüşümlerinin grupları incelenmiş, eşdeğerlik dönüşümlerinin sonsuz küçük üreteçleri, en genel anlamda açık olarak elde edilmiştir. Çalışma, daha önce literatürde bulunan çalışmalardan farklı olarak, lineer olmayan denklemlerin tam çözümlerinin, eşdeğerlik dönüşümleri aracılığıyla elde edilebilir olması üzerine yoğunlaştırılmıştır. Yine daha önce literatürde elde edilmemiş olan, dalga denklemi ailesinin lineer ve lineer olmayan üyeleri arasında mümkün dönüşümlerin üreteçlerinin yapısı üzerine kesin sonuçlar verilmiştir. Bu sonuçlar Teorem $2 ` \mathrm{de}$ özetlenmiştir. Ayrıca denklem ailesinin, alt ailelerinin eşdeğerlik grubu yapısının nasıl incelenebileceği, bir örnek ile verilmiş ve buradan sonuçla, Teorem 3 yazılmıştır.
Son bölümde, eşdeğerlik grubunun üreteçlerinin, lineer ve lineer olmayan denklemler arasında geçişi mümkün kılan bir özel hali örnek olarak ele alınmış ve denklemler arası geçiş ile, lineer olmayan bazı denklemlerin açık çözümü elde edilmiştir. Okuyucu, buna benzer şekilde sadece lineer ve lineer olmayan değil, homojen ve homojen olmayan, sabit katsayılı ile değişken katsayılı vb. denklemler arasında geçişi mümkün kılan dönüşümlerin de üretilebileceğini kolaylıkla görebilir ve uygulayabilir. Bu anlamda Özer in $(2+1)$ boyutlu difüzyon denkleminin eşdeğerlik grupları üzerine olan çalışması [11] incelenebilir.

$\mathrm{Bu}$ çalışma kapsamında elde edilen eşdeğerlik grubunun üreteçleri, denklem ailesinin üyelerinin dönüşümü çerçevesinde ele alınmıştır. Problemin cebrik yapısına değinilmemiştir. Burada açık olarak belirlenmiş olan sonsuz küçük üreteçler kullanılarak, problemin değişmez çözümler araştırılabilir, cebirsel yapı aracılığı ile denklem ailesinin üyeleri arasında sınıflandırma çalışması yapılabilir ki bunların hepsi ayrı birer çalışma konusu olacaktır. .

\section{TEŞEKKÜR}

Çalışmayı, dikkatle okuyup, uyarılarda bulunan hakemlere teşekkürü borç bilirim.

\section{KAYNAKLAR}

[1] De La Rosa, R., Bruzon, M.S. (2018). Differential Invariants Of A Generalized Variable-Coefficient Gardner Equation, Discrete \& Continuous Dynamical Systems - Series S, 11(4), 747-757

[2] Khabirov, S. V. (2018). Group analysis of a one-dimensional model of gas flow in a porous medium. Journal of Applied Mathematics and Mechanics.

[3] Ibragimov, N. H. (2002). Invariants of a remarkable family of nonlinear equations, Nonlinear Dyn., 30, 155-166.

[4] Traciná, R., (2004). Invariants of a family of nonlinear wave equations, Commun Nonlinear Sci Numer Simulat., 9, 127133.

[5] Senthilvelan, M., Torrisi, M., ve Valenti, A. (2006). Equivalence transformations and differential invariants of a generalized nonlinear Schrödinger equation, J. Phys. A: Math. Gen., 9, 3703-3713.

[6] Sophocleous, C., ve Traciná, R. (2008). Differential invariants for quasi-linear and semi-linear wave-type equations, Applied Mathematics and Computation, 202, 216-228.

[7] Huang, D. J., ve Ivanova, N. M. (2016). Algorithmic framework for group analysis of differential equations and its application to generalized Zakharov-Kuznetsov equations. Journal of Differential Equations, 260(3), 2354-2382. 
[8] Bihlo, A., ve Popovych, R. O. (2017). Group classification of linear evolution equations. Journal of Mathematical Analysis and Applications, 448(2), 982-1005.

[9] Long, F. S., Karnbanjong, A., Suriyawichitseranee, A., Grigoriev, Y. N. ve Meleshko, S. V. (2017). Application of a Lie group admitted by a homogeneous equation for group classification of a corresponding inhomogeneous equation. Communications in Nonlinear Science and Numerical Simulation, 48, 350-360.

[10] Jhangeer, A. (2018). Group Classification, Reductions and Exact Solutions of a Class of Higher Order Nonlinear Degenerate Parabolic Equation. International Journal of Applied and Computational Mathematics, 4(1), 2.

[11] Özer, S. (2018). On the Equivalence Groups for (2+1) dimensional Nonlinear Diffusion Equation, Nonlinear Analysis: Real World Applications, No. 43, 155-166.

[12] Huang, D., Zhu, Y., ve Yang, Q. (2016). Reduction operators and exact solutions of variable coefficient nonlinear wave equations with power nonlinearities. Symmetry, 9(1), 3.

[13] Moitsheki, R. J., Hayat, T., ve Malik, M. Y. (2010). Some exact solutions of the fin problem with a power law temperature-dependent thermal conductivity. Nonlinear Analysis: Real World Applications, 11(5), 3287-3294.

[14] Vaneeva, O. (2012). Lie symmetries and exact solutions of variable coefficient $\mathrm{mKdV}$ equations: an equivalence based approach. Communications in Nonlinear Science and Numerical Simulation, 17(2), 611-618.

[15] Şuhubi, E. S. (2008). Diş form analizi. Türkiye Bilimler Akademisi.

[16] Ovsiannikov, L. V. E. (2014). Group analysis of differential equations. Academic Press.

[17] Ibragimov, N. K. (1999). Elementary Lie group analysis and ordinary differential equations (Vol. 197). New York: Wiley.
[18] Lisle, I. (1992). Equivalence transformations for classes of differential equations (Doktora Tezi, University of British Columbia).

[19] Olver, P. J. (2000). Applications of Lie groups to differential equations (Vol. 107). Springer Science \& Business Media.

[20] Lie, S. (1897). Uber Integralinvarianten und ihre Verwertung für die Theorie der Differentialgleichungen, Leipz. Berichte, 49, 369-410.

[21] Şuhubi, E. S. (2000). Explicit determination of isovector fields of equivalence groups for second order balance equations. International journal of engineering science, 38(7), 715-736.

[22] Özer, S. ve Şuhubi, E. (2004). Equivalence transformations for first order balance equations. International journal of engineering science, 42(11-12), 1305-1324.

[23] Şuhubi, E. S. (2004). Equivalence groups for balance equations of arbitrary order-Part I. International journal of engineering science, 42(15-16), 1729-1751.

[24] Şuhubi, E. S. (2005). Explicit determination of isovector fields of equivalence groups for balance equations of arbitrary order-Part II. International journal of engineering science, 43(1-2), 1-15.

[25] Huang, D., Zhu, Y., \& Yang, Q. (2016). Reduction operators and exact solutions of variable coefficient nonlinear wave equations with power nonlinearities. Symmetry, 9(1), 3.

[26] Şuhubi, E. S. (1998). Equivalence transformations for one-dimensional wave equations of balance form. ARI-An International Journal for Physical and Engineering Sciences, 50(3), 151-160.

[27] Harrison, B. K., ve Estabrook, F. B. (1971). Geometric approach to invariance groups and solution of partial differential systems. Journal of Mathematical Physics, 12(4), 653-666.

[28] Cartan, E. (1945). Les systèmes différentiels extérieurs et leurs applications géométriques, Hermann, Paris.

[29] Edelen, D. G. (2005). Applied exterior calculus. Courier Corporation. 